\title{
Multi-System Langerhans cell histiocytosis: Rare case report from Syria
}

\begin{abstract}
Histiocytosis encompasses a group of diverse disorders characterized by the accumulation and infiltration of monocytes, macrophages, and dendritic cells in the affected tissues. The clinical presentations vary greatly, ranging from mild to life threatening. A 46-year-oldman presented to Aleppo University Hospital after 4 years from the beginning of his complaint, because he was living in a hard-to-reach area He presented with grade II dyspnea and painful and swelled legs. Patient past medical history was remarkable for polyuria and polydipsia for 4 years. We diagnosed the patient with multi-organ Langerhans cell Histiocytosis depending on radiological investigations and histopathological examination. We treated the patient with radiation and chemotherapy. On follow up, there was clinical remission but we did not observed any radiological improvement.
\end{abstract}

Keywords: Langerhans cell histiocytosis ( $\mathrm{LCH}$ ), diabetes insipidus, polyuria, pituitary, pulmonary, bone
Volume 9 Issue 4 - 2019

\author{
Ayham Alzahran, ' Ahmad Al-Mouakeh,' \\ Mohammad Ziadeh,' Mohamad Alkhamis,' \\ Osama Mohammad Ibrahim, ${ }^{2}$ MWafa \\ Hamoud Alhussein, ${ }^{3}$ Ziad Aljarad ${ }^{4}$ \\ 'Faculty of Medicine, University of Aleppo, Syria \\ 2Department of Endocrinology, Aleppo University Hospital, Syria \\ ${ }^{3}$ Department of Pathology Aleppo, Ibn Rush Medical center, \\ Syria \\ ${ }^{4}$ Department of Gastroenterology Aleppo University Hospital, \\ Syria
}

Correspondence: Ayham Alzahran, Faculty of Medicine, University of Aleppo, Syria, Tel +963996404250, Email Ayhamzh3@gmail.com

Received: July 17, 2019 | Published: July 26, 2019

\section{Abbreviations: LCH, Langerhans Cell Histiocytosis}

\section{Introduction}

Langerhans cell histiocytosis ( $\mathrm{LCH})$ is a clonal neoplastic disorder characterized by the accumulation and infiltration of dendritic cells in the affected tissue. LCH affects patients from the neonatal period to adulthood and it is more common in children aged $0-15$ years. ${ }^{1}$

$\mathrm{LCH}$ of the bone is a relatively rare disorder of unknown etiology, with an incidence of two cases per million children per year, based upon a comprehensive epidemiologic study conducted by The French National Registry of Childhood Hematopoietic Malignancies. ${ }^{2}$

Histological examination plays a major role in the diagnosis of $\mathrm{LCH}$ as the clinical and imaging characteristics of LCH overlap with several other diseases. ${ }^{3}$

There is no uniform protocol for the treatment of LCH. Therapeutic options for patients with $\mathrm{LCH}$ are surgical resection, radiation therapy, steroid injections, and chemotherapy. Determining the optimal therapy depends on the location and whether it is single or multi organ disease. ${ }^{4}$

\section{Case presentation}

A 46-year-old man presented to Aleppo University Hospital complaining of grade II dyspnea and painful and swelled legs. After a psychological trauma 4 years ago, he started complaining of polyuria and polydipsia (approximately 25-30 litres per day).

He was referred to a general practitioner who prescribed him Desmo press in as a treatment for polyuria and polydipsia without any investigation, because he was living in a conflict zone and he couldn't present to a specialized medical centre due to the war.

The patient's labs level was within normal except for CRP was elevated. By three separated measurement (one hour between each), water deprivation test revealed increased serum osmolality, increased serum $\mathrm{Na}+$ and decreased urine osmolality. Hormonal Laboratory tests showed decreased FT4 and Testosterone. Magnet Resonance Imaging of pituitary gland was normal and no abnormality was detected (Figure1). Chest X-ray showed diffuse interstitial reticular densities in both pulmonary fields (Figure 2). X-ray of pelvis and legs showed lytic lesions.

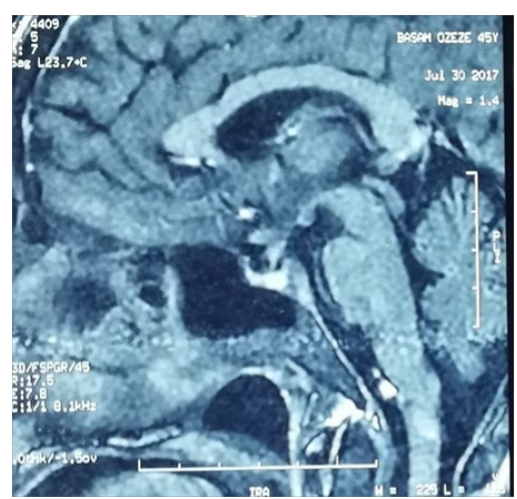

Figure I MRI of Pituitary gland was normal.

Chest Multi-Slice CTscan revealed several diffused interstitial reticular densities in both pulmonary fields with small nodular densities without enlargement of lymphatic nodes (Figure 3). Pelvis MultiSlice CT scan revealed lytic lesions in hip and legs without invasion of adjacent tissues (Figure 4). We sent the Bronchial incisional biopsy taken by bronchoscopy and surgical incisional biopsy taken from left tibia to pathology department.

Pathological report of bronchial biopsy revealed alveolar spaces with infiltration by scattered multinucleated cells, and atypical mononuclear histiocytes with abundant cytoplasm and distinctive folded nuclei with some longitudinal coffee-bean grooves. 
These findings are consistent with Langerhans Cell Histiocytosis (Histiocytosis X)

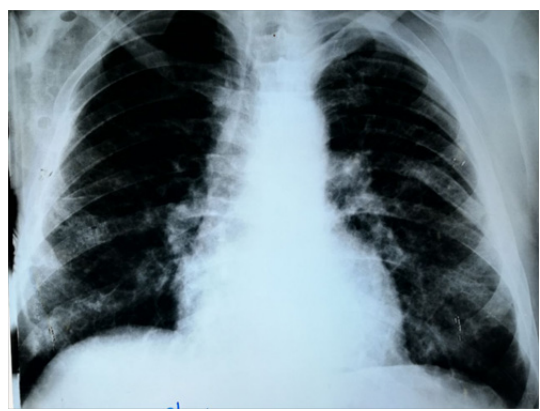

Figure 2 CXR showed diffuse interstitial reticular densities.
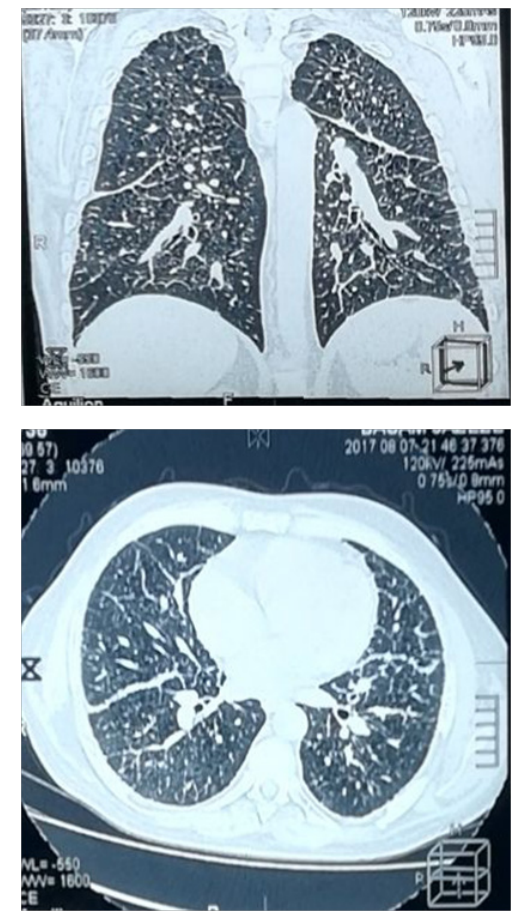

Figure 3 Chest MSCT showing several diffused interstitial reticular densities in both pulmonary fields with small nodular densities.

Pathological report of bone biopsy revealed wide fibrinous, necrotic, and haemorrhagic areas with infiltration of eosinophils, neutrophils, lymphocytes, some multinucleated cells, and atypical mononuclear histiocytes with abundant cytoplasm, and distinctive folded nuclei with some longitudinal coffee-bean grooves. Immunostanis were positive for CD68, S100 and $50 \%$ of diffuse structure positive for KI67. These histological and immunohistochemical findings are consistent with Langerhans Cell Histiocytosis (Histiocytosis X).

Scinticisternography after 3 hours of giving the patient 99MTCMDP showed that there were diffuse osteal hypermetabolic lesions in both humeral bones, both clavicles, both femurs, both tibial bones, both sacroiliac joints, 6th left rib, 7th left rib and 9th right rib (Figure 5). These findings are consistent with neoplastic changes. We treated the patient with Desmopressin, Testosterone and combination of radiation and chemotherapy. On follow up, there was clinical remission but unfortunately, we did not detect any radiological improvement.
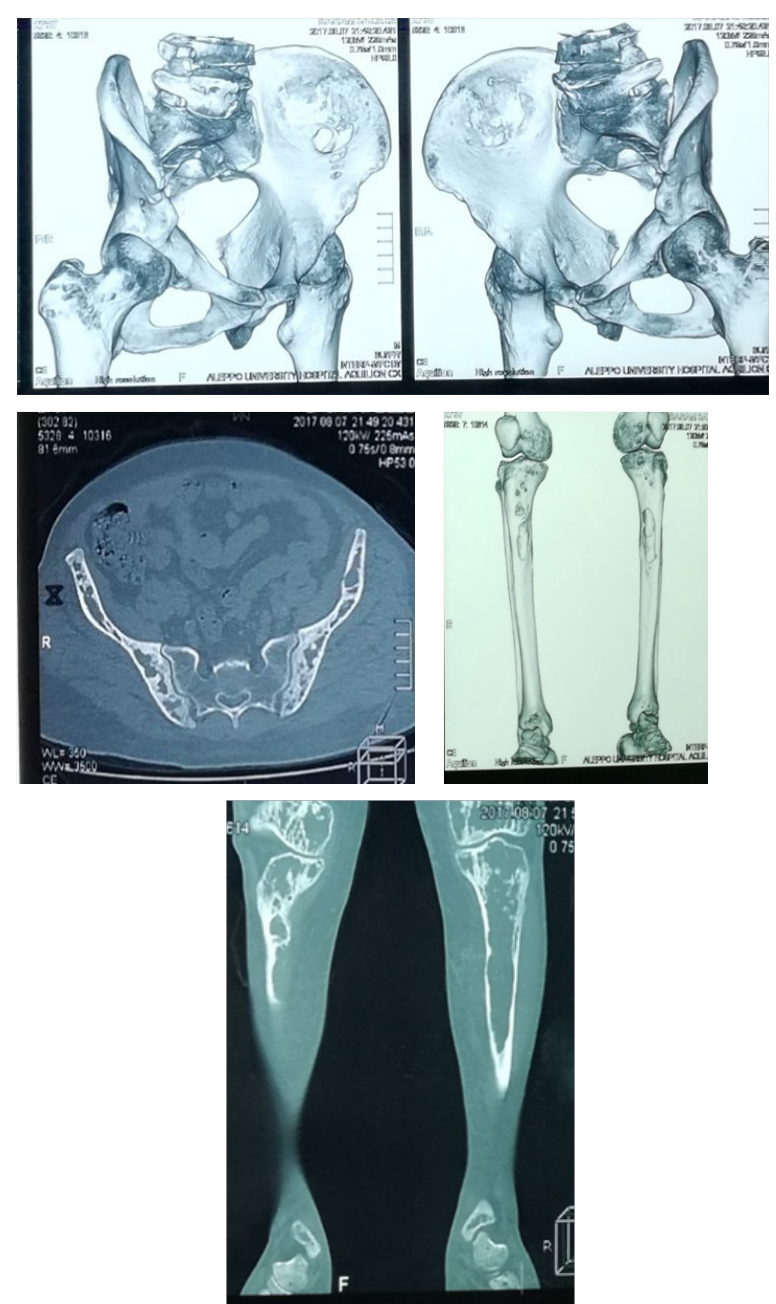

Figure 4 Pelvis Multi-Slice CT scan revealed lytic lesions in hip and legs without invasion of adjacent tissues.

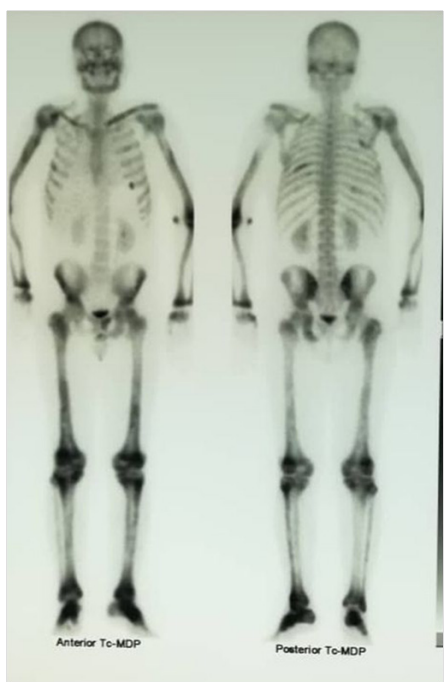

Figure 5 Scinticisternography with ${ }^{99 M}$ TC-MDP showed diffuse osteal hypermetabolic lesions in both humeral bones, both clavicles, both femurs both tibial bones, both sacroiliac joints, $6^{\text {th }}$ left rib, $7^{\text {th }}$ left rib and $9^{\text {th }}$ right rib. 


\section{Discussion}

Langerhans cell histiocytosis (LCH) is a clonal neoplastic disorder characterized by the accumulation and infiltration of dendritic cells in the affected tissue. LCH affects patients from the neonatal period to adulthood and it is more common in children aged 0-15 years. ${ }^{1}$

The acute disseminated multisystem form of LCH usually occurs in children younger than $3 y e a r s$ and has a poor prognosis. The chronic multifocal form of LCH is usually seen in infants and children and it is self-limited in most cases. Unifocal disease commonly manifests in young adults and usually involves the lungs, skin, lymph nodes, pituitary, bone, or GI tract. ${ }^{1}$ In our case, the patient has three organs involved: hypothalamic-pituitary axis, lungs and bones.

Pituitary involvement is more common in systemic disease. The hypothalamic-pituitary axis is rarely a primary site of Langerhans cell histiocytosis involvement. The most common site of CNS involvement in LCH is the hypothalamic-pituitary axis, which results in diabetes insipidus in $10-50 \%$ of patients. ${ }^{2}$ In our case, the initial manifestation was polydipsia and polyuria. Presumptive diagnosis of Diabetes Insipidus was made clinically by giving the patient "desmopressin" due to the inability to do a lab tests and MRI for pituitary gland because the patient was living in a war zone. ${ }^{3}$ years later when the patient could seek Aleppo University Hospital, we performed water Deprivation test and MRI for pituitary gland to confirm the diagnosis.

Hypothalamic disease may also result in other pituitary hormones deficiency, and therefore evaluating endocrine system should be done periodically in all patients diagnosed with $\mathrm{LCH}^{3}$ We evaluated the patient endocrine system and the tests revealed low levels of FT4 and Testosterone.

There is bone involvement in $78 \%$ of patients with $\mathrm{LCH}$. Bone involvement is usually in the flat bones, with lesions of the skull, pelvis, and ribs accounting for more than $50 \%$ of all lesions. The lesions can be painful or asymptomatic. ${ }^{4}$ In our case, the patient presented with pain and edema in both legs and inability to walk.

Between 10 and 20 percent of patients present with infiltration of the oral cavity, usually the posterior mandible. Pain in the jaw and loose teeth may be a presenting symptom. ${ }^{5}$ Our patient also developed teeth loss.

Radiographic features in bone LCH depends on site of involvement and phase of the disease. X-ray of the pelvic and legs showed lytic lesions. MS-CT scan of the pelvic and legs showed several generalized lytic lesions without infiltration to the near tissues. The biopsy from tibia confirmed $\mathrm{LCH}$ diagnosis.

Bone Scinticisternography is useful for detecting any other bone lesions. ${ }^{6}$ After 3 hours of injecting $\left({ }^{99} \mathrm{mTC}-\mathrm{MDP}\right)$ bone scan demonstrated multiple increased activity on both humeral bones, both clavicles, both femurs, both legs, both sacroiliac joints, 6th left rib, 7th left rib and 9th right rib.

Pulmonary involvement is observed in $20-40 \%$ of $\mathrm{LCH}$ patients and can give symptoms, such as pneumothorax, dyspnea, tachypnea, and cough. Imaging studies of the lungs may reveal cysts and micronodular infiltrates. ${ }^{7}$ The patient had grade II dyspnea. CXR and MS- CT imaging of the lungs showed diffused interstitial reticular density. We performed Bronchoscopy and biopsy that showed histological features consistent with LCH.
The diagnosis of LCH should be based on histologic and immunohistochemical examination of biopsies from the lesions. ${ }^{8}$ Pathology report of the biopsy from the left tibia and the lung was positive for immunohistochemical staining of protein markers (CD68) and (S100) confirming the diagnosis of LCH

$\mathrm{LCH}$ responds well to several treatment modalities comprising chemotherapy, surgical excision, steroids or radiation therapy. ${ }^{9-12}$ The patient underwent both radiation and chemotherapy (10 mg of I.V Vinblastine weekly for 5 weeks, then every 2 weeks until now).

The patient showed clinical improvement without any important changes in laboratory or radiographic studies and C-reactive protein level was still elevated which indicate bad prognosis.

\section{Acknowledgments}

None.

\section{Funding}

None.

\section{Conflict of interest}

There is no conflict of interest.

\section{References}

1. Egeler RM, van Halteren AG, Hogendoorn PC, et al. Langerhans cell histiocytosis: fascinating dynamics of the dendritic cell-macrophage lineage. Immunol Rev. 2010;234(1):213-232.

2. Guyot-Goubin A, Donadieu J, Mohamed Barkaoui, et al. Descriptive epidemiology of childhood Langerhans cell histiocytosis in France, 2000 2004. Pediatr Blood Cancer. 2008;51(1):71-75.

3. Ghanem I. Langerhans cell histiocytosis of bone in children and adolescents. J Pediatr Orthop. 2003;23(1):124-130.

4. Plasschaert F, Craig C, Bell R, et al. Eosinophilic granuloma. A different behaviour in children than in adults. J Bone Joint Surg Br. 2002;84(6):870872 .

5. Dunger DB, Broadbent V, Yeoman E, et al. The frequency and natural history of diabetes insipidus in children with Langerhans-cell histiocytosis. N Engl J Med. 1989;321(17):1157-1162.

6. Braunstein GD, Kohler PO. Endocrine manifestations of histiocytosis. Am J Pediatr Hematol Oncol. 1981;3(1):67-75.

7. Stull MA, Kransdorf MJ, Devaney KO. Langerhans cell histiocytosis of bone. Radiographics. 1992;12(4):801-823.

8. Hartman KS. Histiocytosis X: a review of 114 cases with oral involvement. Oral Surg Oral Med Oral Patho. 1980; 49(1):38-54.

9. Dogan AS, Conway JJ, Miller JH, et al. Detection of bone lesions in Langerhans cell histiocytosis: complementary roles of scintigraphy and conventional radiography. J Pediatr Hematol Oncol. 1996;18(1):51-58.

10. Ha SY, Helms P, Fletcher M, et al. Lung involvement in Langerhans' cell histiocytosis: prevalence, clinical features, and outcome. Pediatrics. 1992;89(3):466-469.

11. Lau SK, Chu PG, Weiss LM. Immunohistochemical expression of Langerin in Langerhans cell histiocytosis and non-Langerhans cell histiocytic disorders. Am J Surg Pathol. 2008;32(4):615-619.

12. Allen CE, Mc Clain KL. Langerhans cell histiocytosis: a review of past, current and future therapies. Drugs Today (Barc). 2007;43(9): 627-643. 